\title{
reconnaissance géotechnique depuis une chambre submersible
}

\author{
H.C. VAN DE GRAAF
}

Laboratoire de mécanique des sols de Delft (Pays-Bas)

\section{INTRODUCTION}

La moitié des Pays-Bas se trouve en dessous du niveau de la mer. Ceci explique en partie le développement dans ce pays des méthodes d'opération sousmarines de géotechnique. Ce développement fut accéléré dans les années 70 , visant le marché pétrolier en mer du nord.

L'un des résultats fut le système Mission I, qui comprend une tourelle de plongée, attachée à une plaque de base avec un pénétromètre incorporé. Ce système est la copropriété du laboratoire de mécanique des sols de Delft et de la société Vriens. Il peut être utilisé sous $200 \mathrm{~m}$ d'eau et permet d'effectuer:

- les essais au pénétromètre;

- le carottage Begemann, permettant d'obtenir un seul échantillon intact d'une longueur pouvant atteindre 30 mètres;

- la mesure de la densité en place des sols par méthode électrique ou nucléaire;

- les essais de perméabilité.

Le système Mission II permet également le forage rotatif non destructif.

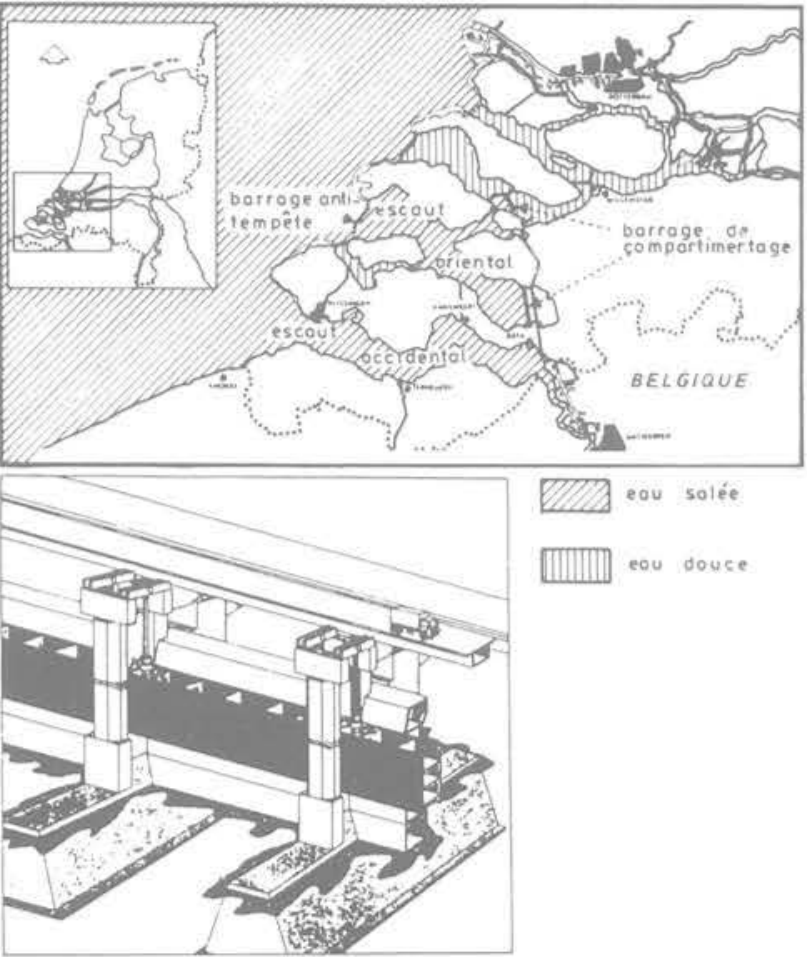

Fig. 1. - Barrage anti-tempête. Situation et vue en perspective. 


\section{LE PLAN DELTA}

Mission I fut employée pour la première fois dans le cadre du plan Delta néerlandais. Le projet d'un barrage anti-tempête d'une longueur de $6 \mathrm{~km}$ dans un terrain de sable meuble exigeait une campagne de reconnaissance extrêmement intensive dans des conditions maritimes difficiles:

- jusqu'à $40 \mathrm{~m}$ d'eau;

- vitesse de courant d'eau $2 \mathrm{~m} / \mathrm{s}$;

- des vagues de $1 \mathrm{~m}$ à $1,5 \mathrm{~m}$ sont très fréquentes.

Une barge spéciale fut construite pour lancer le système Mission.

Le système Mission comprend une plaque de base lourde et une tourelle à partir de laquelle toutes sortes d'essai in situ peuvent être effectués, en sol comme en roche. A cet effet, la plaque de base est équipée d'un vérin creux pour la pénétration des sondes; Mission II est également équipé d'une sondeuse à rotation.

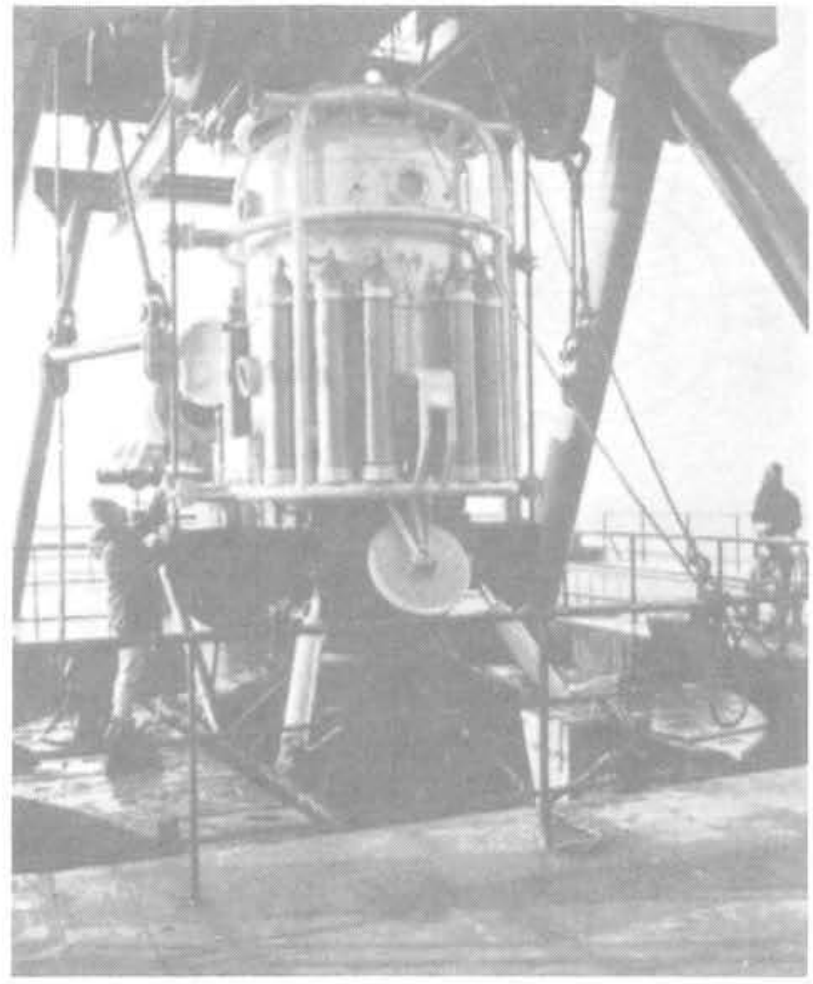

Fig. 2. - Tourelle de plongée Mission.

Bien que le système ait été conçu pour résister à des pressions à $200 \mathrm{~m}$ de profondeur, la pression dans la tourelle n'est qu'atmosphérique pour la plupart des essais. Dans certains cas, lors d'un carottage par exemple, cette pression est la même que la pression ambiante. A cet effet, Mission comporte un système de saturation.

Le premier problème fut la détermination de la zone à compacter par vibroflotation par les moyens suivants:
- au pénétromètre statique à pointe électrique (quelques centaines);

-. par carottage Begemann (quelques dizaines);

- par pénétrométrie utilisant les sondes densitométriques (quelques dizaines).

Ces deux dernières méthodes furent développées à Delft dans les années 60 . La densitométrie électrique se fait au moyen de 2 sondes pénétrométriques. La première sonde permet de mesurer la résistance à la pointe, le frottement latéral, et la résistance électrique du sol. La deuxième sonde permet de mesurer la résistivité électrique de l'eau interstitielle. Au moyen d'un étalonnage au laboratoire, on détermine, pour un terrain donné le rapport entre le quotient résistivité eau/résistivité sol et la porosité.

Cette méthode, aujourd'hui couramment utilisêe pour les terrains sableux, fut employée fréquemment dans la deuxième partie de l'opération avec comme objectif l'optimalisation du compactage par vibroflotation.

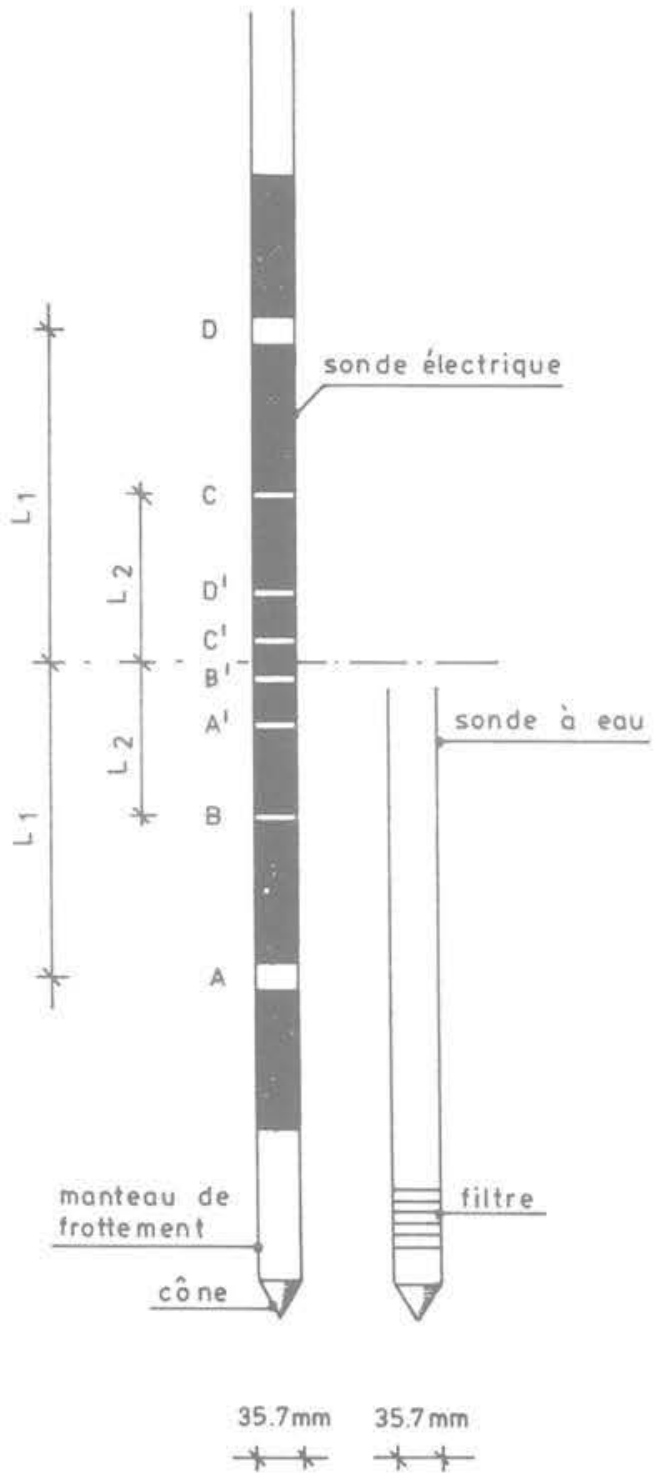

Fig. 3. - Sondes utilisées pour la mesure électrique de la densité. 


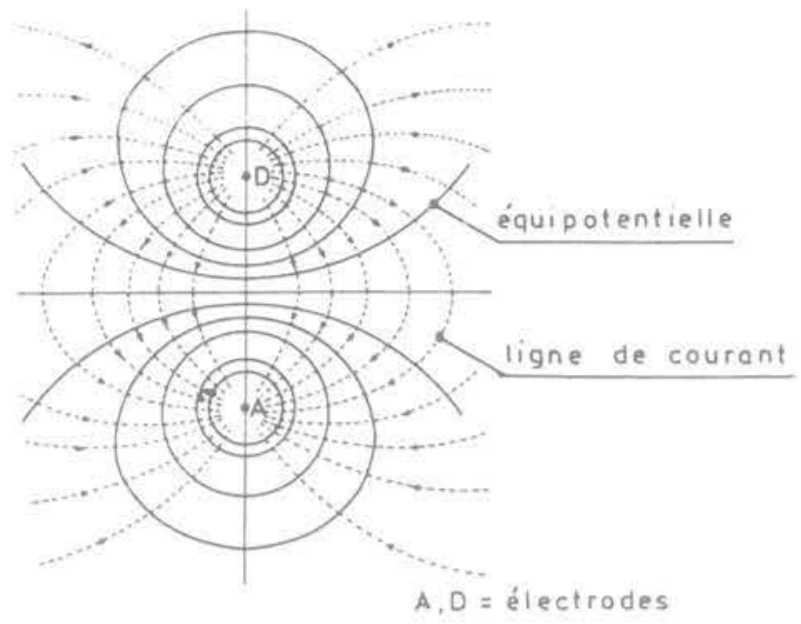

Fig. 4. - Système de lignes de courant et d'équipotentielles engendré par une différence de potentiel entre $A$ et $D$.

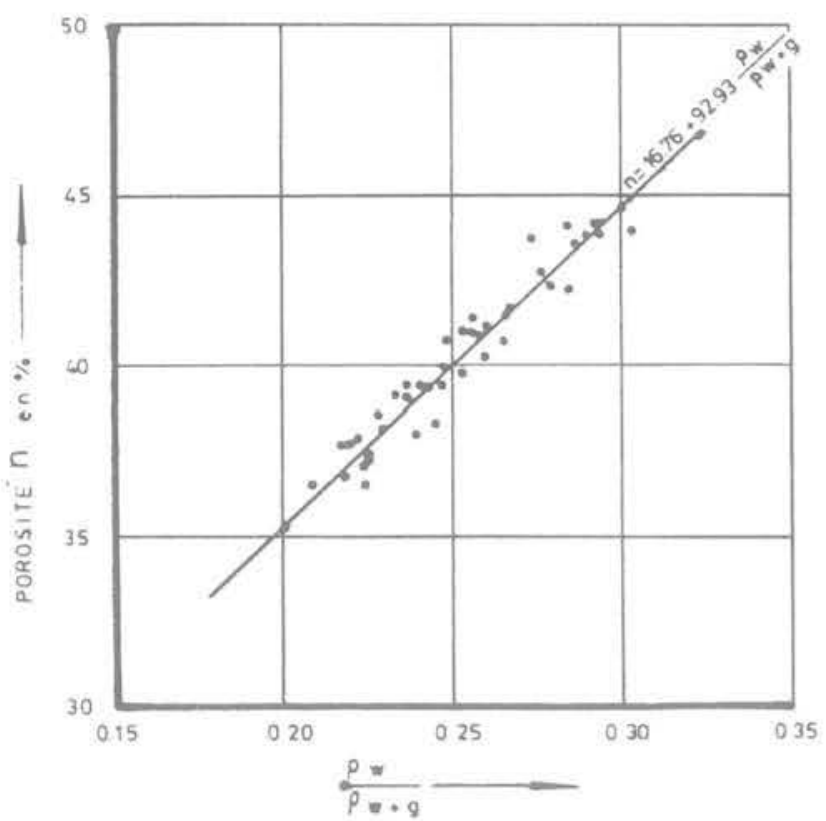

Fig. 5. - Courbe d'étalonnage.

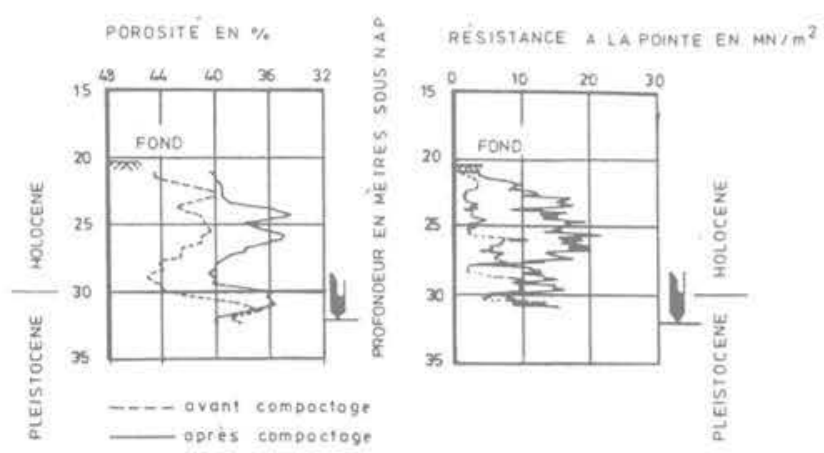

Fig. 6. - Résistance à la pointe et porosité avant et après compactage.

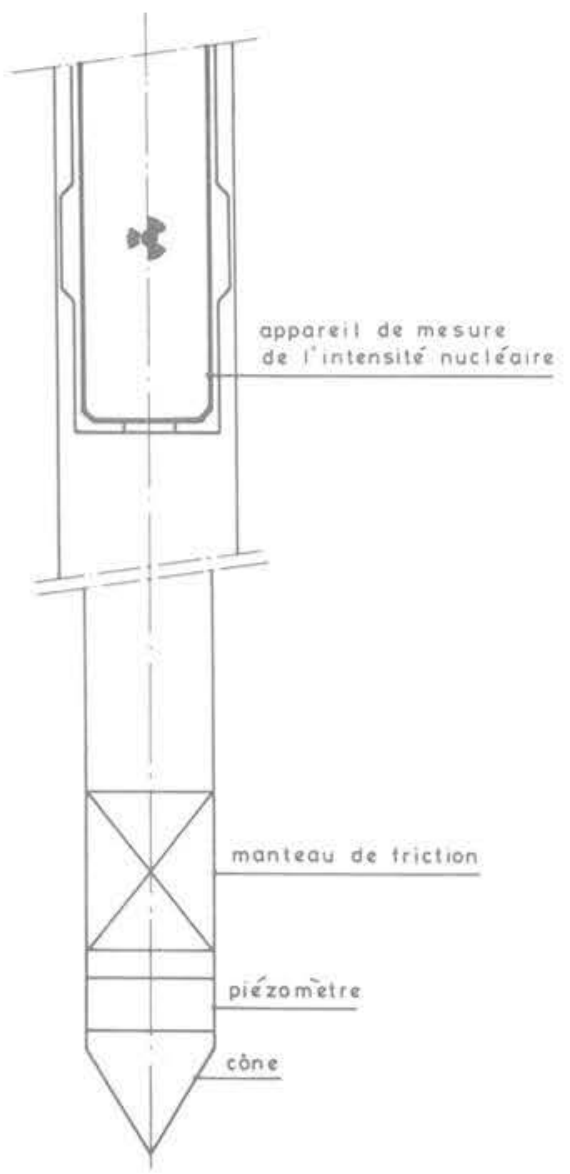

Fig. 7. - Appareil de mesure de densité nucléaire.

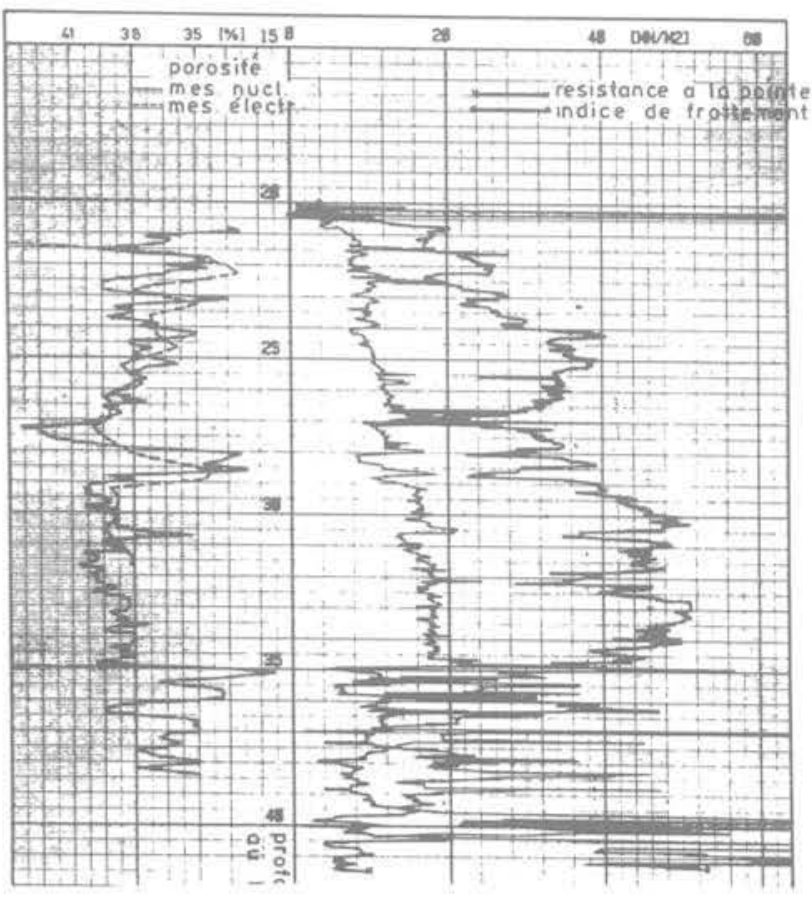

Fig. 8.

A gauche: comparaison entre les résultats de mesures électriques et nucléaires.

A droite: résultats de la pénétrométrie. 


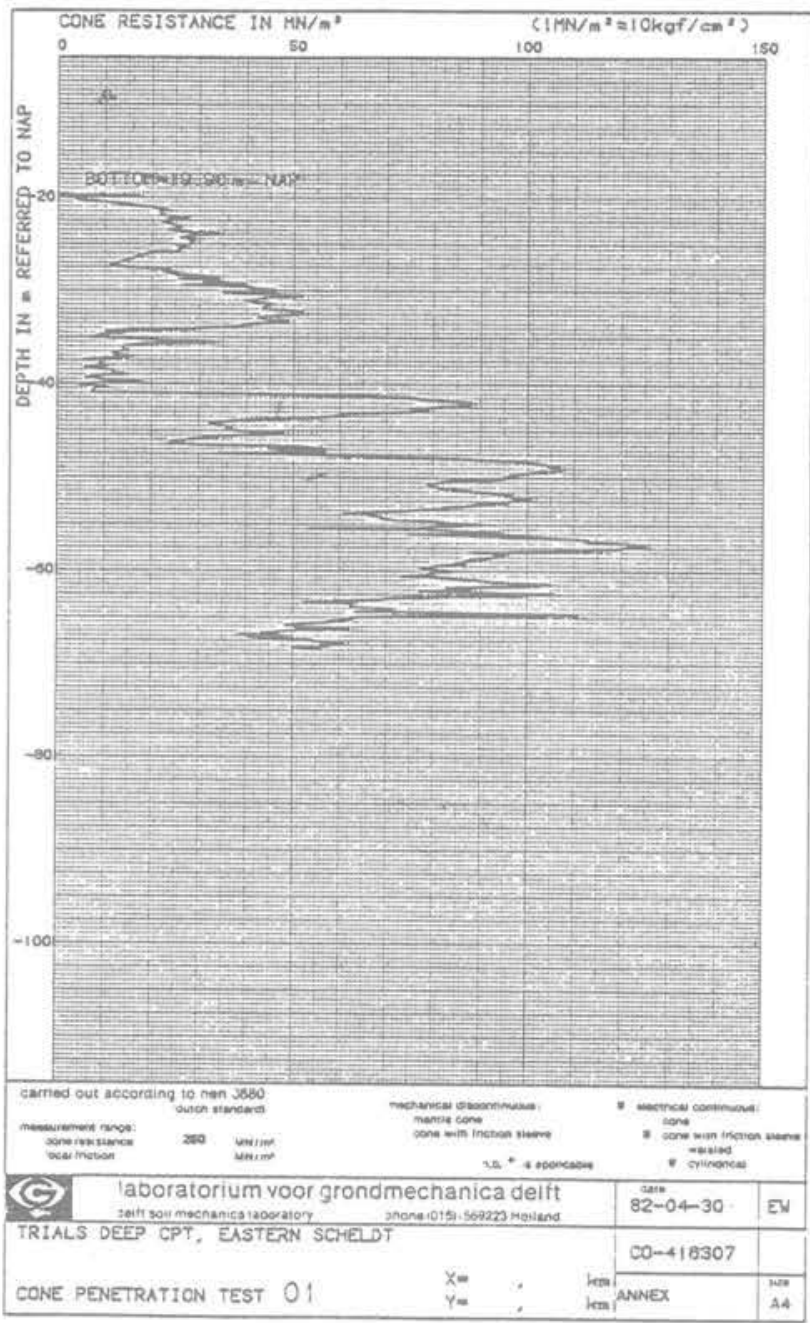

Fig. 9. - Résistance au cône en fonction de la profondeur.

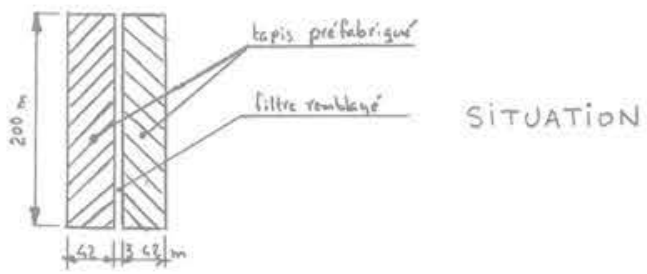

Fig. 10. - Situation.

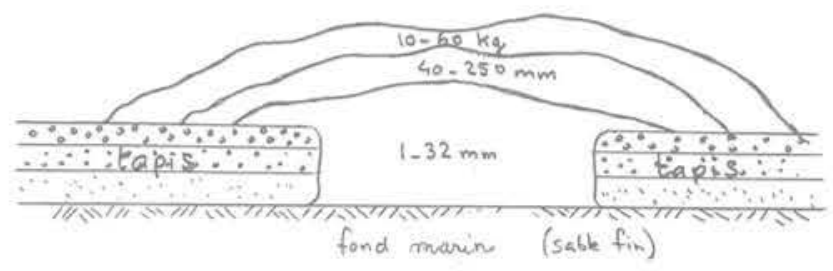

Fig. 11. - Coupe transversale du remblai.

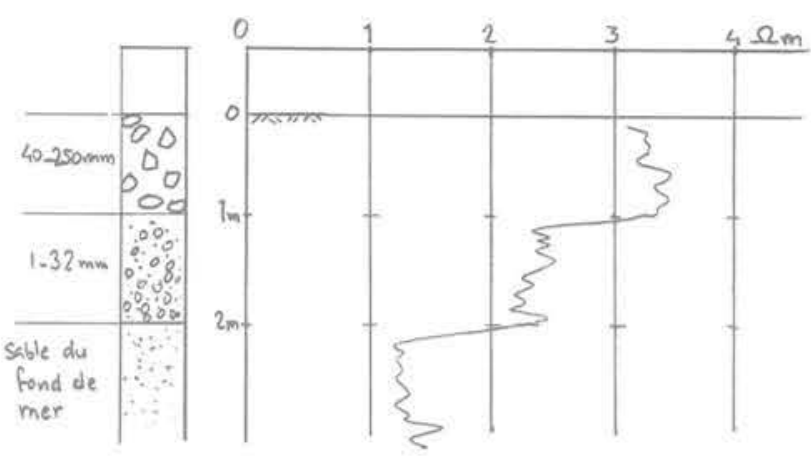

Fig. 12, - Résistivité électrique du sol.

L'inconvénient de cette méthode d'une qualité excellente par ailleurs est que l'utilisation de la *sonde à eau demande beaucoup de temps dans un terrain moins perméable comme le sable fin ou limoneux. C'est pour cette raison qu'à été développée la densitométrie nucléaire par pénétrométrie. Cette méthode comprend un essai pénétrométrique électrique normal, à l'exception du diamètre de la pointe et des tiges, qui est de $45 \mathrm{~mm}$ au lieu de $36 \mathrm{~mm}$. Après avoir atteint le refus on retire le cable électrique et on descend à l'intérieur des tiges jusqu'à la pointe le train de mesure nucléaire comprenant une source rayons $Y$ de 3 millicurie et un appareil de mesure. Ensuite on remonte le train de tiges, y compris le train de mesure tout en effectuant les mesures de la densité. L'avantage c'est qu'ainsi le risque de perte de la source nucléaire durant l'effort de pénétration est évité.

Le résultat de la mêthode nucléaire est comparable à celuî de la méthode électrique.

Pour augmenter la profondeur de pénétration, on a réalisé une pointe d'un diamètre légèrement plus grand que celui des tiges et d'une qualité d'acier beaucoup plus résistant, ceci dans le but de diminuer le frottement latéral, et utilisé une deuxième tige qui empêche le flambage de la première et qui diminue son frottement.

L'essai suivant a êté réalisé en appliquant une force de $600 \mathrm{kN}$ sur une tige de $36 \mathrm{~mm}$.

Actuellement, les travaux de compactage de la barge Mytilus sont terminés mais d'autres problèmes géotechniques se posent, en particulier, le problème du filtre.

Les piliers du barrage seront placés sur ce filtre, qui doit éviter que dans le cas d'un barrage fermé, l'eau qui passe sous le barrage et qui remonte ensuite vers le fond emporte les grains de sable. Le filtre devra garantir que l'eau puisse sortir du fond et non pas le sable. De plus ce filtre doit résister à l'érosion par le courant dans le cas d'un barrage ouvert.

Ce filtre est constitué par des tapis préfabriqués, composés de trois couches de sable grossier ou gravier fin. Entre deux tapis, on construit le filtre sur place par remblayage sous l'eau. 
Actuellement Mission I est utilisé pour contrôler la composition de ce filtre au moyen de carottage Begemann ou par la mesure de la résistivité électrique du sol. Ce paramètre est une bonne indication pour la détermination de la composition d'une couche.

En dehors du système Mission, un autre système est utilisé pour ces essais. C'est un appareil téléguidé, nommé Geodoff, qui permet la pénétrométrie statique légère $(30 \mathrm{kN})$ ou le vibrocarottage.

Geodoff fut employé également en Mer du Nord pour la reconnaissance du tracé d'un pipe-line.

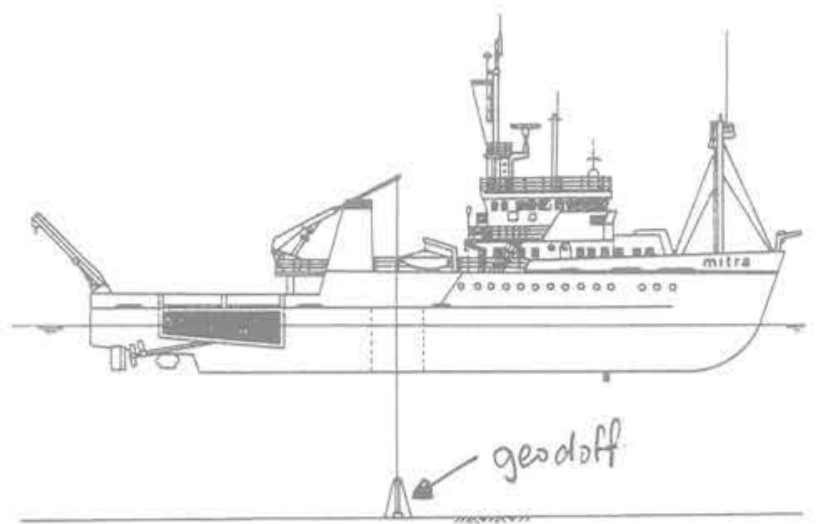

Fig. 13. - Le système Geodoff.

Comme vous voyez le système Mission I a toujours été occupé (soit opérationnel, soit paré) dans le Oosterschelde entre 1976 et 1983.

Son succès a conduit à la construction de Mission II, muni en supplément d'une foreuse à rotation, employé en 1981 à Terreneuve.

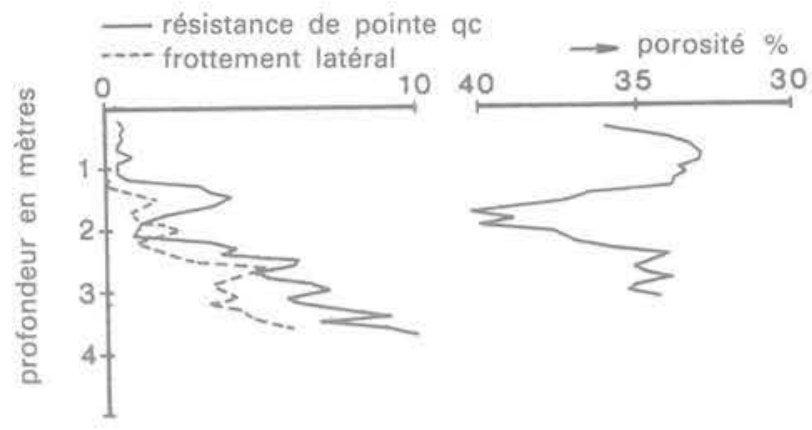

Fig. 14. - Essai d'intensité électrique le long du pipe-line.

\section{UNE APPLICATION DU SYSTEMME MISSION II AU CANADA}

Le projet de pose d'un cable électrique entre le Labrador et Terreneuve dans le détroit de Belle-Isle nécessite la réalisation d'une tranchée dans le fond de la mer qui le protège contre les icebergs qui heurtent le fond. Au fond, le sol est constitué de quelques mètres de sable, gravier et pierres reposant sur la roche saine (calcaire ou grès).

Les conditions nautiques sont extrêmement difficiles: 110 mètres d'eau, une vitesse du courant jusqu'à $2 \mathrm{~m} / \mathrm{s}$, un vent qui arrive fréquemment à 7 Beaufort, même en bonne saison, causant une houle de 2 à 3 mètres. De plus il faut tenir compte du fait que les opérations seront interrompues par le passage d'icebergs.

En 1976 on avait tenté sans succès d'obtenir des carottes suivant là méthode traditionnelle depuis un

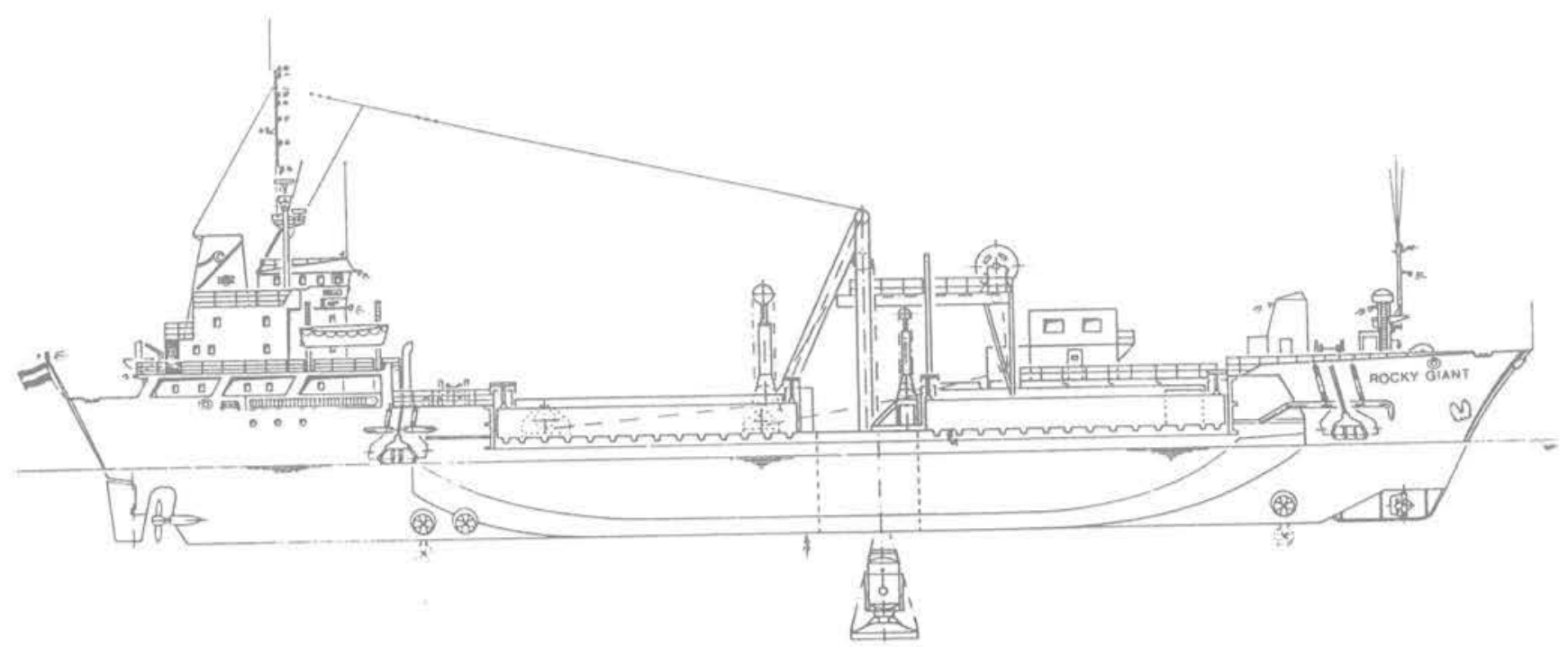

Fig. 15. - Le bateau "Rocky Giant" équipé de Geodoff. 
bateau. On a considéré que, dans ces conditions, seul un système submersible serait en mesure d'obtenir de bons résultats. Le système Mission II fut sélectionné en 1981 et équipé pour cette opération d'une foreuse à rotation. On a utilisé les carottiers Nassovia (carotte $\varnothing$ $105 \mathrm{~mm}$ ) et Longyear HQ (62 mm).

Le bateau «Rocky Giant» fut positionné dynamiquement avec des ancres comme système de secours. Mission fut lancé dans la piscine au centre du bateau.

Au cours d'un mois on a réalisé 17 trous, soit au total 88 mètres, dont $42 \mathrm{~m}$ dans la roche.

\section{BIBLIOGRAPHIE}

1. DE LEUW, E.H. : SILENCE, P. - Contrôle in situ du compactage de remblais sous eau - Bulletin de l'Association Internationale de Géologie de l'Ingénieur, no. 26-27, Paris 1983.
2. VAN DE GRAAF, H.C.; SMITS A.P. - Offshore site investigation by rotary drilling from a diving bell - Ground Engineering, January 1983.

3. Brochure. - Density measurements in situ and critical density: Laboratoire de Mécanique des Sols de Delft.

4. NIEUWENHUIS, J.K.; SMITS, F.P. - The development of a nuclear probe in a cone penetrometer. - Comptes rendus de ESOPT-Il 1982, Amsterdam, Volume 2, 745-749.

5. VERMEIDEN, J. - The submersible working chamber - Delft Soil Mechanics Laboratory, 1977.

6. WAKELING, T.R.M. - The Mission system, site investigation from a diving bell. - Offshore Services, 1980.

7. SMITS, A.P. - Mission II, now equipped for all seabed investigations. - Second Canadian Conference in Marine Geotechnical Engineering, 1982. 